\title{
Radical Cystectomy in the Octogenarian Population: A Single Centre Experience
}

\author{
Omer A. Raheem Mohamed H. Kamel Peter Leung William P. Shields \\ Stephen S. Connolly Antonio J. Zimmerman \\ Ponnusammy Mohan \\ David P. Hickey \\ Department of Urology, Beaumont Hospital, Dublin, Ireland
}

\author{
Key Words \\ Radical cystectomy • Octogenarian
}

\begin{abstract}
Introduction: Radical cystectomy is the treatment of choice for infiltrating non-metastatic bladder cancer. However there is reluctance to perform this surgery in the elderly due to morbidities associated. We sought to examine our institutional experience regarding radical cystectomy in the octogenarians. Patients and Methods: A total of 23 were 80 years or older in age (19 males and 4 females). Overall mortality, early and late morbidity were analyzed. Length of hospital stay, intensive therapy unit admissions, re-hospitalization and overall survival were assessed. Results: Median age at time of cystectomy was 83.5 years (range 80-90 years). Median follow-up time was 7.5 years (range 8-120 months). All patients had standard radical cystectomy. Median operative time was 3 hours (range 3-5 hours). Median number of blood transfusion units was 9 (range 4-14). Median preoperative and postoperative hemoglobin level was 12.5 (range 9.9-14) and $10.8 \mathrm{~g} / \mathrm{dl}(8.7-12.2 \mathrm{~g} / \mathrm{dl}$ ) respectively. Admission to the intensive therapy unit was needed in 3 patients. Median number of hospitalization days following the cystectomy procedure was 23 days (range 5-90 days). Median survival time was 16 months and the actuarial survival at 1,2 and 3 year was $66.7,47.6$ and $12 \%$ respectively. Conclusion: This study highlights the safety and feasibility of radical cystectomy in the treatment of bladder cancer in the octogenarians. It provides palliation of local symptoms and local cancer
\end{abstract}

\section{KARGER}

Fax +4161306 1234

E-Mail karger@karger.ch

www.karger.com
(C) 2011 S. Karger AG, Basel

$1015-9770 / 11 / 0054-0196 \$ 26.00 / 0$

Accessible online at:

www.karger.com/cur control. Future research should be directed towards comparing with the non-octogenarian cohorts with particular emphasis on oncological, functional and survival outcomes as end points.

Copyright @ 2011 S. Karger AG, Basel

\section{Introduction}

The European population is ageing and the current life expectancy of females and males born now is 87 and 83 years respectively. The increased life expectancy over the last few decades in most areas of the world has greatly increased cancer burden in the elderly [1].

Bladder cancer is the fifth leading cause of cancer death in the 8th decade [1]. The incidence of bladder cancer has significantly increased over the last 20 years. It is now more than $160 / 100,000$ in people over 75 years old [2]. Currently, radical cystectomy is the treatment of choice for muscle invasive bladder cancer [2]. However there is reluctance to perform this surgery in the elderly due to complications associated.

We aimed to examine our institutional experience regarding radical cystectomy in the octogenarians, with particular emphasis on the clinical and surgical managements as well as oncological outcomes. 
Table 1. Patients' demographics and details

\begin{tabular}{lcc}
\hline & $\mathrm{n}$ & $\%$ \\
\hline Patient & 23 & \\
$\begin{array}{l}\text { Median age (range), years } \\
\text { Sex }\end{array}$ & $83.5(80-90)$ & \\
$\quad$ Male & 19 & 82.6 \\
$\quad$ Female & 4 & 17.4 \\
ASA & & \\
$\quad$ Class III & 23 & 100 \\
Pathology T stage & & 70 \\
$\quad$ < pT2 & 16 & 30 \\
$\quad$ pT3 & 7 & 87 \\
Pathological grade & & 13 \\
$\quad$ High grade & 20 & \\
$\quad$ Low grade & 3 & \\
\hline
\end{tabular}

\section{Patients and Methods}

\section{Selection of Patients}

Between January 11996 and December 31 2008, 102 radical cystectomies were performed in our department. Of these patients 23 were 80 years or older in age. We attempted to perform simple procedure with no extensive lymph node dissection and reconstructed urinary diversion as ileal conduit. Patient demographics and details are illustrated in table 1. Patients were composed of 19 males and 4 females. Median age at time of cystectomy was 83.5 years (range 80-90 years). Assessment of existing co-morbid conditions and indications of cystectomy was performed (table 2). Overall mortality, early (within 90 days after surgery) and late complications (more than 90 days after surgery) were assessed. Length of hospital stay, intensive therapy unit (ITU) admissions, re-hospitalization, overall survival and causes of death were assessed.

\section{Preoperative Assessment}

All patients had extensive history taking and physical examination. Symptoms of heart disease as dyspnea, chest pain and syncope were carefully evaluated. Known risk factors to cardiac complications as smoking, diabetes mellitus and renal failure were assessed for presence and severity. Assessment of pulmonary status with attention to the presence of chronic obstructive pulmonary disease, long history of smoking or abnormal chest examination was done. American society of anesthesiologists (ASA) physical status classification was employed and all patients were assigned ASA class $3(100 \%)$. General anesthesia was recommended and subsequently performed in all patients.

Assessment of anticoagulation status included careful history taking of antiplatelets or warfarin medications and performing coagulation screen. Prophylaxis against deep venous thrombosis was performed by the use of elastic stockings and low molecular weight heparin. All patients had assessment of their nutritional status especially those with recent weight loss. Cognitive status
Table 2. List of existing co-morbid condition in patients

\begin{tabular}{|c|c|}
\hline System & Number \\
\hline \multicolumn{2}{|c|}{ Concomitant bladder cancer related complications } \\
\hline Bleeding bladder & 5 \\
\hline \multicolumn{2}{|l|}{ Hydronephrosis } \\
\hline -Unilateral & 1 \\
\hline -Bilateral & 1 \\
\hline \multicolumn{2}{|l|}{ Respiratory } \\
\hline Chronic obstructive airways disease & 5 \\
\hline Asthma & 1 \\
\hline \multicolumn{2}{|l|}{ Cardiovascular } \\
\hline Hypertension & 8 \\
\hline Ischemic heart disease & 7 \\
\hline Hyperlipidemia & 4 \\
\hline Myocardial infarction & 3 \\
\hline Atrial fibrillation & 2 \\
\hline Sick sinus syndrome & 1 \\
\hline Congestive heart failure & 1 \\
\hline Abdominal aortic aneurysm $(4.27 \mathrm{~cm})$ & 1 \\
\hline \multicolumn{2}{|l|}{ Endocrine } \\
\hline Hyperthyroidism & 1 \\
\hline \multicolumn{2}{|l|}{ Gastrointestinal } \\
\hline Peptic ulcer disease & 3 \\
\hline Diverticulitis & 2 \\
\hline Chronic colitis & 1 \\
\hline Epigastric hernia & 1 \\
\hline Gastro-esophageal reflux & 1 \\
\hline Vitamin B12 deficiency & 1 \\
\hline \multicolumn{2}{|l|}{ Neurological } \\
\hline Depression & 4 \\
\hline Parkinson's disease & 3 \\
\hline Cerebro-vascular accident & 1 \\
\hline Transient ischemia attack & 1 \\
\hline Seizure & 1 \\
\hline Trigeminal neuralgia & 1 \\
\hline Deafness & 1 \\
\hline \multicolumn{2}{|l|}{ Rheumatological } \\
\hline Osteoarthritis & 3 \\
\hline \multicolumn{2}{|l|}{ Dermatological } \\
\hline Psoriasis & 1 \\
\hline Basal cell carcinoma of scalp & 2 \\
\hline Actinic keratosis & 1 \\
\hline
\end{tabular}

assessment was usually performed on patient admission by nursing staff. All patients were visited by the stoma nurse who marked the optimal site for the stoma and explained the stoma care to the patient. This helped to reduce patient anxiety as regards urinary diversion.

In the preoperative evaluation, particular emphasis was made to make the patient familiar with the procedure and supportive therapies; this helped to reduce the fear of the unknown and improved performance. We believe this should be done in conjunction of supportive family member. Routine laboratory tests included complete blood count, urea and electrolytes, serum al- 
bumin, chest X-ray and electrocardiogram. In addition, computerized tomography of chest, abdomen and pelvis was performed in all patients for disease staging prior to surgical intervention and revealed no evidence of distant metastasis.

\section{Postoperative Management}

Patients were nursed in the ward in a high dependency unit with continuous vital data monitoring. It is not the routine to admit patients to ITU unless medically indicated. Adequate pain control was achieved with epidural analgesia. Nasogastric tube to decompress the stomach was usually inserted postoperatively. All patients received postoperative chest physiotherapy and incentive spirometry. Parenteral antibiotic prophylaxis started at the time of induction of anesthesia and continued for 1-3 days postoperatively. Preoperative anticoagulation was continued postoperatively till the patient was fully ambulant. Patients were allowed to start eating soft diet on 4th to 6th postoperative day. Patients were encouraged to ambulate on the second postoperative date.

\section{Postoperative Follow-Up}

Postoperatively, patients were routinely seen 3 weeks after discharge then at 3 monthly intervals during the first year, 6 monthly intervals during the second year then annually thereafter. Patients were assessed clinically as well as blood tests for electrolytes and creatinine levels, base excess and ultrasound of the kidneys were performed.

\section{Results}

Median follow-up time was 7.5 years (range 8 months10 years). None of our patients had neoadjuvant and/or adjuvant treatment. Median operative time was 3 hours (range 3-5 hours). Median number of blood transfusion units was 9 (range 4-14). Median preoperative hemoglobin level was $12.5 \mathrm{~g} / \mathrm{dl}$ (range $9.9-14 \mathrm{~g} / \mathrm{dl}$ ). Median postoperative hemoglobin level is $10.8 \mathrm{~g} / \mathrm{dl}$ (range 8.7-12.2 $\mathrm{g} / \mathrm{dl}$ ). Admission to the ITU was needed in 3 patients, each one stayed only over the night in ITU. The median survival time was 16 months and the actuarial survival at 1,2 and 3 year was $66.7,47.6$ and $12 \%$ respectively. Causes of death are illustrated in table 3 .

\section{Early Complications}

Details of postoperative complications are listed in table 4 . Early postoperative complications were divided into minor and major. There were $14(52 \%)$ early minor postoperative complications which were successfully responded to conservative measures. Another 11 ( $41 \%)$ early major postoperative complications was observed in patients. One patient developed adhesive intestinal obstruction and had bowel resection, subsequently he developed paracolic abscess that was drained under computerized tomography guidance but developed gram
Table 3. Causes of death in patients

\begin{tabular}{ll}
\hline Cause of death & Number \\
\hline Medical related causes & \\
Cardiac problems & 2 \\
Respiratory problems & 2 \\
Septicaemia & 2 \\
Stroke & 1 \\
Renal failure & 0 \\
Cancer related causes & \\
Local or distant recurrences & 1 \\
Other malignancies & 0 \\
Unknown & 0 \\
\hline
\end{tabular}

Table 4. Early and late postoperative complications

\begin{tabular}{lcc}
\hline Complication & Number & $\%$ \\
\hline Early minor complications & 14 & 52 \\
Atelectasis & 4 & \\
Confusion & 5 & \\
Dehydration & 1 & \\
Atrial fibrillation & 3 & \\
Paralytic ileus & 1 & \\
Early major complications & 1 & \\
Enteric fistula & 2 & \\
Rectourethral fistula & 1 & \\
Urine leakage & 1 & \\
Adhesive intestinal obstruction & 1 & \\
Right paracolic abscess & 1 & \\
Pneumonia & 2 & \\
Methicillin-resistant staphylococcus aureus & 1 & \\
Myocardial infarction & 1 & \\
Stroke & 1 & \\
Late complications & 2 & \\
Stomal stenosis & 1 & \\
Metabolic acidosis & 1 & \\
\hline
\end{tabular}

negative septicemia and died 1 month after the operation. Pneumonia developed in 2 additional patients. One of the two developed respiratory failure as a complication of pneumonia and died 1 month after the operation while the other responded to medical treatment. Enteric fistula occurred in 2 patients. In 1 patient the fistula was successfully managed with total parenteral nutrition. In the other patient, successful operative repair was done and the patient survived for 13 months following the proce- 
dure. One patient had local recurrence in the pelvis presenting as recto-urethral fistula. He was managed with sigmoid colostomy but he developed gram negative septicemia and died 3 months later. One patient developed stroke secondary to atrial fibrillation. He had reasonable recovery following physiotherapy and is currently alive and doing well. Methicillin-resistant staphylococcus auerus infection occurred in one patient and was managed by isolation and antibiotics till 3 negative swabs were obtained. The only fatal heart complication in this study occurred in 1 patient who developed myocardial infarction in the immediate postoperative period and died.

\section{Late Complications}

Two (7\%) late postoperative complications (> 90 days) developed. One patient developed stomal stenosis and had his ileal conduit stoma refashioned. In another patient, dehydration and acidosis as a complication of the urinary diversion occurred and was managed conservatively.

\section{Hospital Length of Stay}

Median number of hospitalization days following the cystectomy procedure was 23 days (range 5-90 days). Re-hospitalization was needed in only 2 patients. One patient had refashioning of ileal conduit stoma and the other was admitted for correction of dehydration and acidosis.

\section{Discussion}

Europe is one of the major regions of the world where population ageing is most advanced. The median age of the population in Europe is higher than in all other parts of the world. The proportion of older persons (i.e. 60 years and older) in 1998 was higher in Europe (20.3\%) than in North America (16.2\%) and in all other continents [3-5]. Age alone is not an important determinant of cancer risk, but rather a surrogate of prolonged carcinogen exposure. It is estimated that one-half of all older persons will undergo surgery at some time during the remainder of their lives. As a consequence, the nature of surgical practice is changing as a result of changing population demographics. The median age on most general surgical services now exceeds what was once considered a contraindication to operate. The bulk of morbidity and mortality related to surgery in the elderly derives from the cardio-respiratory systems. Fifty percent of mortality alone results from cardiovascular complications [6].

Radical Cystectomy in the Octogenarian Population
In addition to the public health problems, it has to be noted that the deal of clinical issues affecting elderly cancer patients sometimes makes it difficult to apply diagnostic and therapeutic standardized protocols. Moreover, the problem to adopt treatment with curative intent often is largely due to co-morbid conditions other than cancer and physiological impairment [7]. As the percentage of ageing population increased, the number of people suffering from bladder cancer increased as well. In the United States it was estimated that 54,200 new cases of bladder cancer would be diagnosed in 1999 with 12,000 projected deaths from the disease [8].

As in most European countries, in France the incidence rate of bladder cancer increased $25 \%$ in men and 9\% in women between 1975 and 1995 [9]. Radical cystectomy may cure an otherwise lethal bladder cancer and provides a radical treatment to symptoms of bladder cancer, many of these are very difficult to tolerate in the frail octogenarian [10]. However such operation is a major undertaking for the elderly patient.

In this study, a reasonable median survival time of 16 months was achieved despite the old age of our patients (median age 83.5 years). The 1- and 2-year survival rates were 66.7 and $47.6 \%$ respectively. This is similar to the results of Stroumbakis et al. [11]. Although complications occurred more frequently in the early postoperative period, the majority of postoperative complications were manageable.

Both Gamé et al. [12] and Lance et al. [13] reported approximately $63 \%$ incidence of early postoperative complications in a similar cohort of octogenarians. Chang et al. [14] reported a much lower postoperative morbidity, $28 \%$ in a selected cohort of high risk elderly patients. We believe the reason for that was a routine postoperative ITU admission to all of his high risk patients. The most common minor early complication in this study was confusion (5 patients), all of them recovered. Delirium is a common complication in surgical patients, occurring in $10-15 \%$ of all ages and often in older patients. Studies of elderly patients with hip fractures have shown that up to $61 \%$ develop postoperative confusion [15]. Causes of delirium are multiple. It can often be the presenting manifestation of medical illness such as pneumonia, urinary tract infection, or myocardial infarction. It may also be caused by metabolic abnormalities, overload or a variety of medications especially those with anticholinergic properties. Withdrawal from alcohol or psychoactive drugs may also cause delirium [16].

We believe the best approach to treatment of delirium is trying to prevent it. This can be achieved by keeping

Curr Urol 2011;5:196-201 
the patient oriented by verbal and visual cues like ensuring they have their glasses and or hearing aids, also keeping medications to a minimum, and good medical care to avoid medical problems is mandatory. We had 2 (7\%) late complications. An 83-year-old female patient who had had stomal stenosis managed by refashioning and had an otherwise uneventful postoperative follow-up and survived for 14 months after the cystectomy procedure. The second patient was a 90 years old male patient who was admitted 12 months after the procedure with dehydration as a complication of the urinary diversion and was managed conservatively. This patient survived for 30 months following the cystectomy procedure. The incidence of late postoperative complications we had in this study was similar to the results reported by Figueroa et al. [10].

Median hospital stay was 23 days. This reflects the difficulty of managing postoperative complications in the elderly. However many of the studies that report lower number of hospitalization days in the octogenarian were performed in the United States [11-14]. Caution must be made in comparing our results with these studies since the health system in Europe is different from that in the US. We had a low re-hospitalization rate with only 2 patients requiring hospital readmission. Median operative time was 3 hours. The reason was that we did not perform extensive lymph node dissection and we adopted simple urinary diversion. The median number of intraoperative blood transfusion units was 4. Certainly in this study, the long-term survival was not a realistic goal in such very old patients but rather relief of bladder cancer symptoms and improved quality of life for the remaining short life span for those patients.

We reviewed the literature as regards other treatment modalities that had been used in elderly bladder cancer patients. Radiation treatment had been tried in elderly patients with bladder cancer. In a study by Holmang et al. [17], high incidence (47\%) of radiation side effects was noticed with none of these side effects improving. Adding to this, there was $23 \%$ re-hospitalization rate and $5 \%$ mortality rate. Salminen [18] reviewed 155 patients with urinary bladder cancer who were not suitable for radical surgery, treated with full-course radiotherapy, 6,600 cGy over 9-weeks period, split-course. He reported recurrences in 94 patients $(60 \%)$ with $16 \%$ of his patients developing reduced bladder capacity and worsening of irritative bladder symptoms.

Zietman et al. [19] evaluated urodynamic findings, bowel and bladder symptoms following an organ preserving protocol composed of tri-modality treatment of transurethral resection, chemotherapy and radiotherapy in a questionnaire sent to 32 patients. He reported a low compliant bladder in 7 patients $(21.8 \%)$ with urinary incontinence in $19 \%$ of patients, abdominal cramps in $13 \%$ and difficult bowel control in $22 \%$. Chemotherapy had been tried in elderly patients with bladder cancer. However the prerequisite of a good performance status to start such treatment is usually difficult to achieve in these patients with multiple co-morbid conditions. In addition, neutropenia is a serious complication following chemotherapy. There is a demonstrated correlation between neutropenic complications and age with life threatening infections in up to $40 \%$ of elderly patients receiving chemotherapy for cancer [20]. Goffin et al. [21] reported on 14 elderly bladder cancer patients, evaluated tolerance of elderly patients to radiation treatment and chemotherapy. Mild toxicity was reported in all patients with $6(42 \%)$ developing grade 3 or 4 toxicity. Von der Maase et al. [22] published the results of a large randomized, multicenter phase 3 study comparing gemcitabine and cisplastin to the traditional methotrexate, vinblastine, doxorubicin and cisplatin (MVAC) used in the treatment of advanced bladder cancer. High incidence of toxicity was noticed in the gemcitabine and cisplastin arm with grade 3-4 neutropenia and anemia in 71 and $27 \%$ respectively and gastrointestinal toxicity in $26.5 \%$. In the methotrexate, vinblastine, doxorubicin and cisplatin arm, toxicity was worse with severe neutropenia in $82 \%$, neutropenic fever in $14 \%$, mucositis in $22 \%$ of patients with the need to give a granulocyte-colony stimulating factors as well as prophylactic antibiotics, antiviral and antifungal medications.

\section{Conclusion}

The findings of this study demonstrate the safety and feasibility of radical cystectomy and urinary diversion in the treatment of bladder cancer in the octogenarians. We stress on preoperative optimization of co-morbid conditions, short operative time, the use of simple urinary diversion and aggressive postoperative care for the success of the operation. Adding to this, radical cystectomy provides palliation of local symptoms, local cancer control and long-term survival benefits might be expected after surgery, especially in patients with organ-confined disease. 


\section{References}

1 European Commission: Eurostat statistics in focus, population and social conditions. European Commission, vol 94, Paris, 2005.

$>2$ Boring CC, Squires TS, Tong T, Montgomery S: Cancer statistics, 1994. CA Cancer J Clin 1994;44:7-26.

3 Berlie J: Epidemiologie du cancer chez les personnes agees. Oncologica 1992;4:9-14.

$\checkmark 4$ Gschwend JE, Fair WR, Vieweg J: Radical cystectomy for invasive bladder cancer: contemporary results and remaining controversies. Eur Urol 2000;38:121-130.

5 United Nations population division: World population prospects - the 2000 revision, vol I-III. New York, 2001.

6 Hackford AW: Surgical principles for the aged: care of the elderly; in Reichel W, Gallo JJ, Whitehead JB, Delfs JR, Murphy JB (eds): Clinical aspects of aging, ed 4. Philadelphia, Lippincott Williams and Wilkins, 2001, pp408-415.

$>7$ Fentiman IS, Tirelli U, Monfardini S, Schneider M, Festen J, Cognetti F, Aapro MS: Cancer in the elderly: why so badly treated. Lancet 1990;335:1020-1022.

$>8$ Landis SH, Murray T, Bolden S, Wingo PA: Cancer statistics, 1999. CA Cancer J Clin 1999;49:8-31.

$>9$ Ménégoz F, Black RJ, Arveux P, Magne V, Ferlay J, Buémi A, Carli PM, Chapelain G, Faivre J, Gignoux M, Grosclaude P, MaceLesec'h J, Raverdy N, Schaffer P: Cancer incidence and mortality in France in 1975-95. Eur J Cancer Prev 1997;6:442-466.
10 Figueroa AJ, Stein JP, Dickinson M, Skinner EC, Thangathurai D, Mikhail MS, Boyd SD, Lieskovsky G, Skinner DG: Radical cystectomy for elderly patients with bladder carcinoma: an updated experience with 404 patients. Cancer 1998;83:141-147.

11 Stroumbakis N, Herr HW, Cookson MS, Fair WR: Radical cystectomy in the octogenarian. J Urol 1997;158:2113-2117.

12 Gamé X, Soulié M, Seguin P, Vazzoler N, Tollon C, Pontonnier F, Plante P: Radical cystectomy in patients older than 75 years: assessment of morbidity and mortality. Eur Urol 2001;39:525-529.

13 Lance RS, Dinney CP, Swanson D, Babaian RJ, Pisters LL, Palmer LJ, Grossman HB: Radical cystectomy for invasive bladder cancer in the octogenarian. Oncol Rep 2001;8: 723-726.

14 Chang SS, Alberts G, Cookson MS, Smith JA: Radical cystectomy is safe in elderly patients at high risk. J Urol 2001;166:938-941.

15 Gustafson Y, Brannstrom B, Norberg A, Bucht $\mathrm{G}$, Winblad B: Underdiagnosis and poor documentation of acute confusional states in elderly hip fracture patients. J Am Geriatr Soc 1991;39:760-765.

16 Snustad DG, Lindsay RW: Perioperative assessment and management in older patients; in Evans JG, Williams TF (eds): Oxford textbook of geriatric medicine, ed 4. Oxford, Oxford University Press,1992, pp674-682.
17 Holmang S, Hedelin H, Borghede G, Johansson SL: Long-term followup of a bladder carcinoma cohort: questionable value of radical radiotherapy. J Urol 1997;157:1642-1646.

18 Salminen E: Recurrence and treatment of urinary bladder cancer after failure in radiotherapy. Cancer 1990;66:2341-2345.

19 Zietman AL, Sacco D, Skowronski U, Gomery P, Kaufman DS, Clark JA, Talcott JA, Shipley WU: Organ conservation in invasive bladder cancer by transurethral resection, chemotherapy and radiation: results of a urodynamic and quality of life study on longterm survivors. J Urol 2003;170:1772-1776.

20 Monfardini S, Aapro M: Cancer treatment in the elderly: the need for a better organization. Ann Oncol 2007;18:1283-1284.

21 Goffin JR, Rajan R, Souhami L: Tolerance of radiotherapy and chemotherapy in elderly patients with bladder cancer. Am J Clin Oncol 2004;27:172-177.

22 von der Maase H, Hansen SW, Roberts JT, Dogliotti L, Oliver T, Moore MJ, Bodrogi I, Albers P, Knuth A, Lippert CM, Kerbrat P, Sanchez Rovira P, Wersall P, Cleall SP, Roychowdhury DF, Tomlin I, Visseren-Grul CM, Conte PF: Gemcitabine and cisplatin versus methotrexate, vinblastine, doxorubicin, and cisplatin in advanced or metastatic bladder cancer: results of a large, randomized, multinational, multicenter, phase III study. J Clin Oncol 2000;18:3068-3077. 